\title{
Using PDMS Micro-Transfer Moulding for Polymer Flip Chip Packaging on MEMS
}

\author{
Edward K.L. Chan, Cell K.Y. Wong, M. Lee, Matthew M.F. Yuen, Yi-Kuen Lee \\ Department of Mechanical Engineering \\ Hong Kong University of Science and Technology \\ Clear Water Bay, Kowloon, Hong Kong SAR, China \\ Email: edwardc@ust.hk, cacell@ust.hk, leolee@ust.hk,meymf@ust.hk,meyklee@ust.hk
}

\begin{abstract}
Polymer flip chip process utilizes Ag filled thermoset and/or thermoplastic polymers, in combination with stencil printing processes to form polymer bump interconnects of flip chip I.C. devices [1]. Nevertheless, slumping behavior of conventional polymer interconnect material during bumping process on the wafer chip has constrained the bump height achieved by the most common stencil printing to $50 \mu \mathrm{m}[2]$. A bumping process to form fine pitch, high aspect ratio polymer flip chip bumps without slumping was developed by producing a through-hole PDMS micro-transfer mould on a metallized wafer [3]. In this paper, a MEMS chip [4] was packaged by the PDMS micro-transfer mould technique to evaluate the performance of the interconnection method. Flip chip assembly process was then performed on the printed circuit board (PCB) and some functionality tests was conducted afterwards. The designed dimension of the polymer bump is $150 \mu \mathrm{m}$ in diameter, $90 \mu \mathrm{m}$ in height, and at a $245 \mu \mathrm{m}$ pitch. Bump adhesion force was measured and found to be about 40gram. Electrical conductivity test was also conducted after PCB assembly as preliminary reliability test of the whole assembly process. Results showed that the MEMS chip was functioning well with new polymer bumping method. With this bumping approach, intrinsic slumping problem of the conductive polymer paste is resolved. This low temperature fabrication technique is an important development for MEMS packaging.
\end{abstract}

\section{Introduction}

Amongst different types of chip-level interconnections, the flip chip provides the highest packaging density and performance and the lowest packaging profile. A flip chip is defined as a chip directly mounted on a substrate with the chip surface facing the substrate using metallic or polymer bumps on the wafer as the interconnecting path. The flip chip technology is developed in view of the demanding performance requirements of high density packages. Its rapid application has fostered research on improving the flip chip technology using different materials or methods [5].

Amongst the flip chip interconnecting techniques, the three basic technologies are anisotropic materials, inorganic metallic bump technology, and isotropic conductive polymers. Conventional flip chip uses solder as the interconnection material. However, it needs high processing temperature, and requires high capital equipment investment. Polymer flip chip process utilizes $\mathrm{Ag}$ filled thermoset and/or thermoplastic polymers in conjunction with stencil printing processes to form polymer flip chip devices [1]. Micro-electro-mechanical systems (MEMS) or micro-opto-electro-mechanical systems (MOEMS) [6] requires low temperature fabrication techniques which is particularly suited for polymer flip chip applications. With polymer flip chip, direct interconnection using conductive bumps merges level $1 \& 2$ interconnects to realize maximum space conservation, high package density, short conducting path, low lead inductance, high assembly yield and high performance. Various polymer flip chip techniques have been developed in recent years. Nevertheless, slumping behavior of conventional polymer interconnect material during the stencil printing wafer bumping process has constrained the bump height to $50 \mu \mathrm{m}$ [2]. It does not meet the pitch size and bump geometry requirements for typical flip chip packages. This restricts the application of polymer flip chip bumping process.

A bumping process to form fine pitch, high aspect ratio polymer flip chip bumps without slumping was developed by producing a through-hole polydimethylsiloxane (PDMS) micro-transfer mould $(\mu \mathrm{TM})$ on a metallized wafer [3]. From previous studies, the packaging was limited to simple silicon wafer with simple circuit and the polymer bumps did not have a good wetting with the gold pad due to the high aspect ratio of the PDMS holes [3]. In this paper, a MEMS chip [4] was packaged by the PDMS micro-transfer mould technique to evaluate the performance of the interconnection method. Process flow chart of polymer bumping by PDMS microtransfer mould is shown in following diagram.

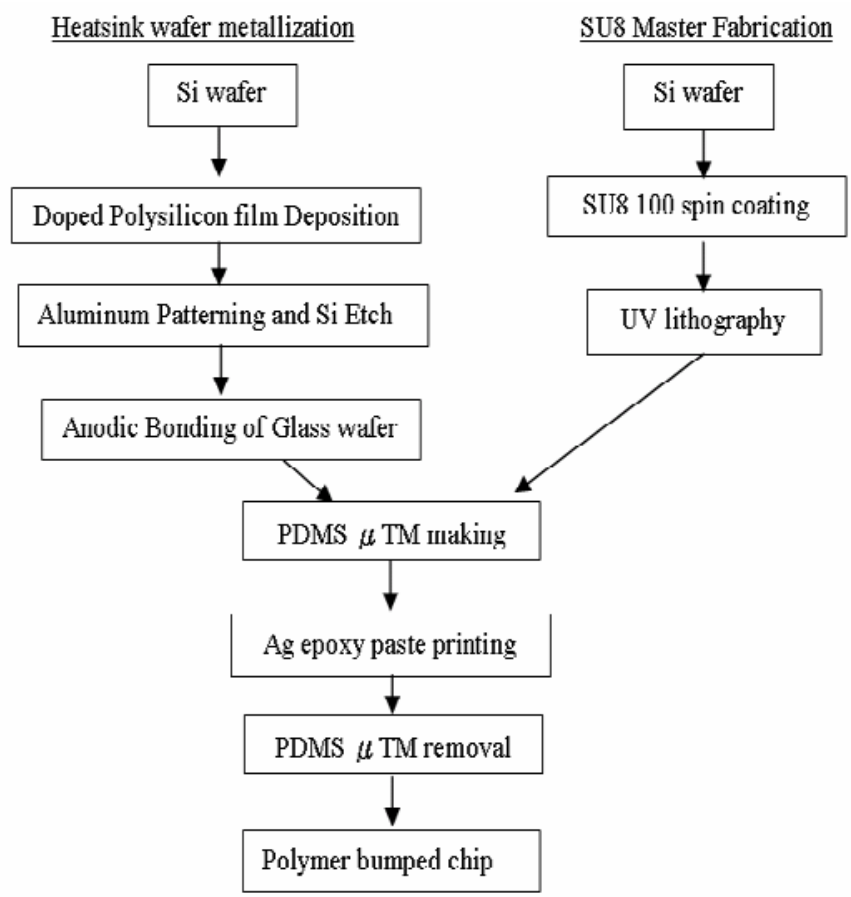

Figure 1 Process flow of polymer bumping technique by PDMS $\mu$ TM technique 


\section{MEMS Chip}

The MEMS Chips used this study were provided by Lee $e t$ al [4]. The high aspect ratio micro heat sink consisted of microchannels, an integrated local heater and a temperature microsensor array was used to study the flow patterns and thermal performance of the microsystem. The overall footprint of a single die was about $12 \mathrm{~mm} \times 23 \mathrm{~mm}$. The MEMS chip had two substrates, a glass substrate and a silicon substrate which were bonded by anodic bonding. The glass substrate had one inlet hole and one outlet hole for the fluid running through the microchannel between two substrates. The chips had $144 \mathrm{I} / \mathrm{Os}$ with a pitch of $245 \mu \mathrm{m}$ on the silicon substrate. Each pairs of I/Os were connected to a temperature sensor which was made of phosphorus doped polysilicon. A schematic cross-section of the microchannel heat sink and its picture is shown in Figs $.1 \& 2$ respectively.

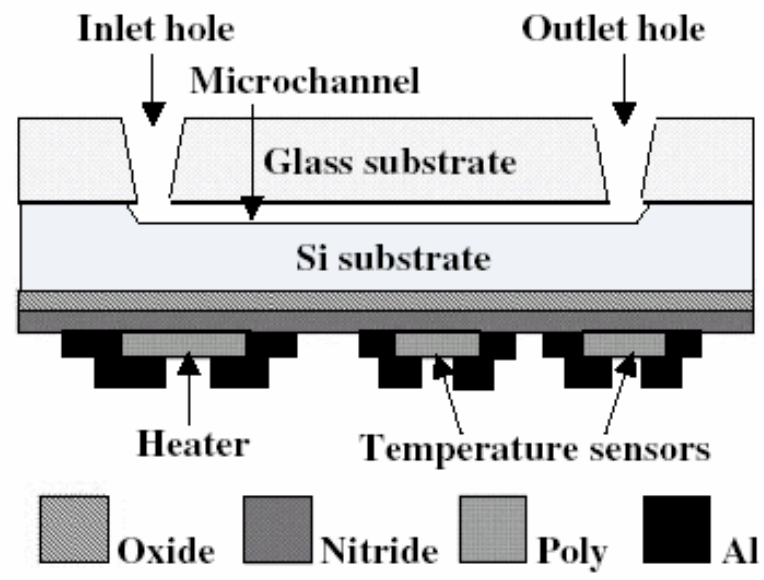

Figure 2 A schematic cross-section of microchannel heat sink (Lee et al) [4]

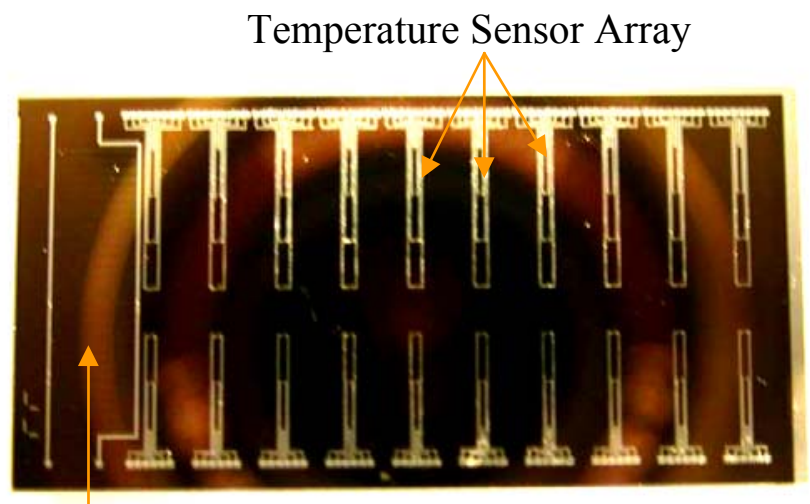

Heater

Figure 3 Picture of a fabricated micro heat sink showing the heater, temperature sensors and Al metal lines.

\section{PDMS $\boldsymbol{\mu T M}$ Fabrication}

A through-hole PDMS mould was first fabricated on the MEMS chip using the following procedure. Silicon wafer with patterned negative photoresist, SU-8 100 (Microchem, Inc.; Newton; Midland, MI), was fabricated as a master wafer for the PDMS micro-transfer mould. The silicon wafer was deposited with a layer of $120 \mu \mathrm{m}$ thick SU-8 negative photoresist by spin coating at 2500rpm. The SU8 master mask with the same pattern as the I/Os of MEMS chip was aligned on the top and the SU-8 pattern was formed in 25 seconds under UV exposure. A post exposure bake (PEB) was conducted at $90^{\circ} \mathrm{C}$ to release the stress in the polymer. Subsequently, the SU- 8 was removed by developing the wafer in the SU8 developer.

The SU-8 master wafer was then aligned with the patterned MEMS chip and high pressure bonding was performed by a Toray Flip Chip Bonder. The liquid PDMS was introduced to flow between the two wafers and curing was conducted under high pressure $(101.239 \mathrm{~N})$. A PDMS mould was formed after curing at $80^{\circ} \mathrm{C}$ for one and half hour.

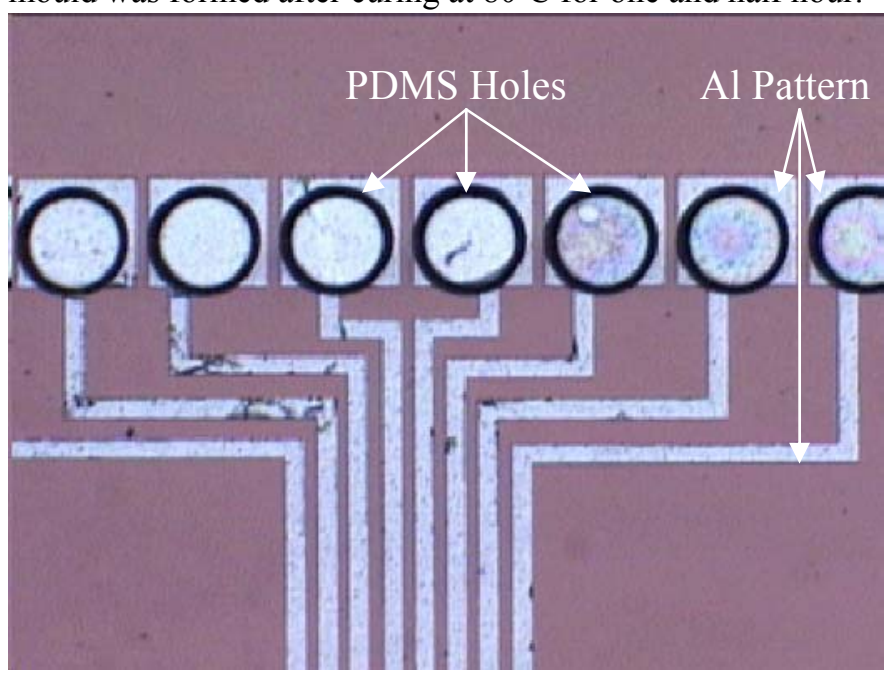

Figure 4 PDMS $\mu$ TM with through holes (100x)

\section{Polymer Bump Fabrication}

Wong et al [3] suggested that the adhesion of the polymer bumps on the pads is mainly due to the wetting of the bumps with the I/O pads. The conductive polymer printing into the PDMS holes was performed manually resulting in poor control of the thickness of the epoxy residue on top of the PDMS mould. Furthermore, the process is adversely affected by the intrinsic viscosity and wetting behavior of the silver paste resulting in incompletely filled holes in the PDMS mould. The air trapped inside the small cavity also hindered the filling and led to inconsistent results. To resolve these problems, two process modification steps were introduced. Wetting of polymer bumps and bond pads was improved by conducting the epoxy filling process in vacuum to enhance the integrity of the polymer bumps. A print tool with a tilted knife was also introduced to enhance the even spreading of epoxy over the PDMS stencil. The print tool was placed inside a vacuum chamber. The whole printing process was performed under a vacuum environment. 


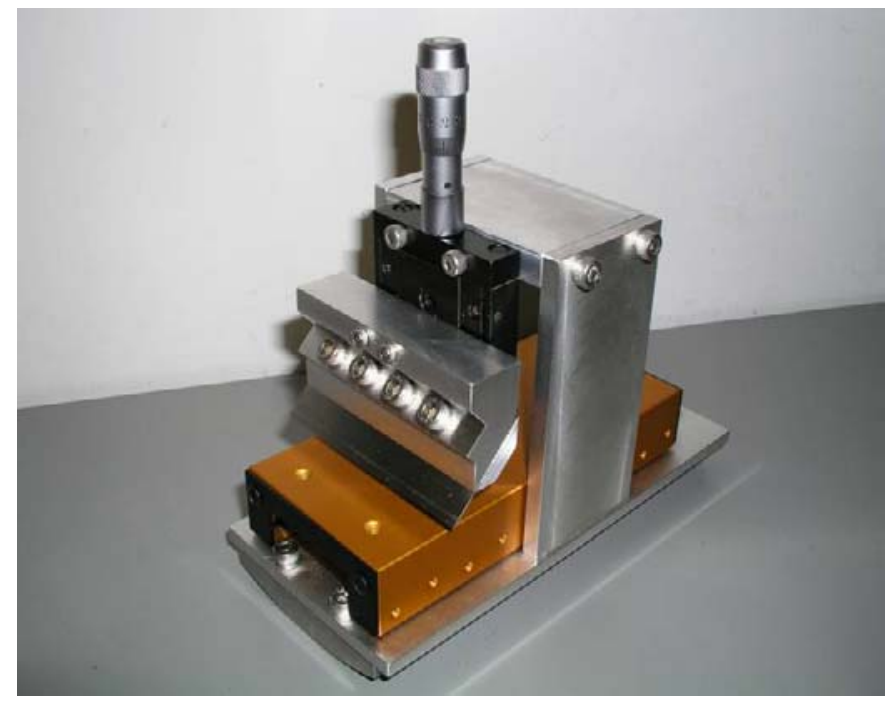

Figure 5 Print tool

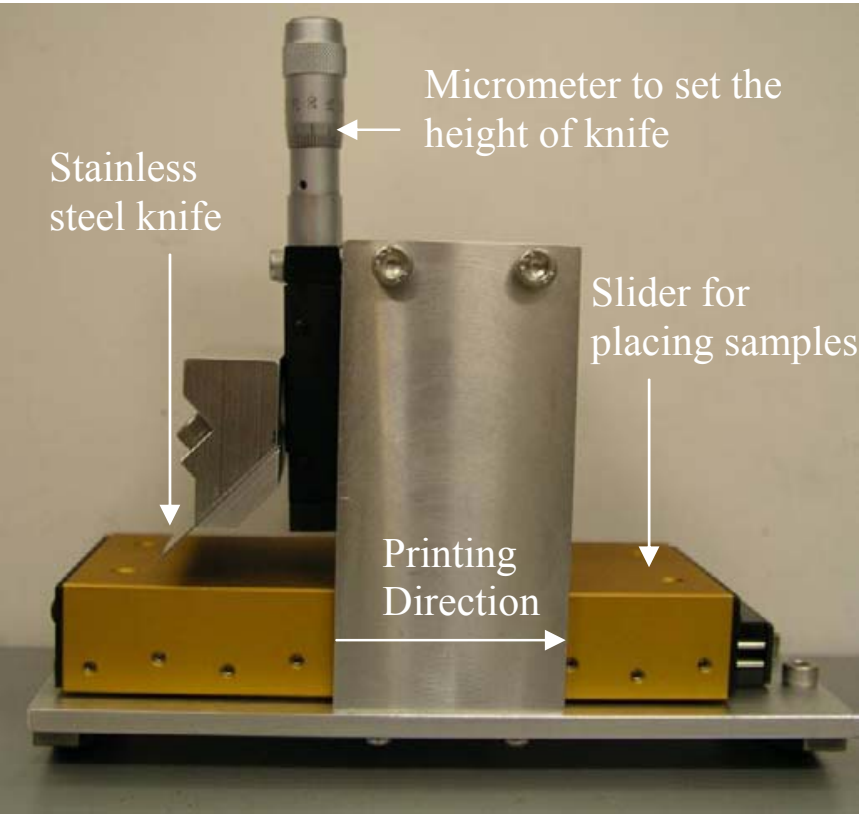

Figure 6 Print tool mechanism

Silver filled conductive polymer (EPO-TEK H20E-PFC) was used to fill the PDMS through holes. The printing knife was set at $15 \mu \mathrm{m}$ above of the PDMS mould surface. The vacuum level was set to 0.1 Bar. After printing, the sample was then cured at $80^{\circ} \mathrm{C}$ for one and a half hours. The PDMS micro-transfer mold was then dissolved by tetrabutylammonium fluoride (TBAF) (Aldrich, Milwaukee, $\mathrm{WI}$ ) in $1 \mathrm{M}$ Tetrahydrofuran (THF). Isopropyl alcohol and deionized water were used in rinsing to remove the TBAF residue.

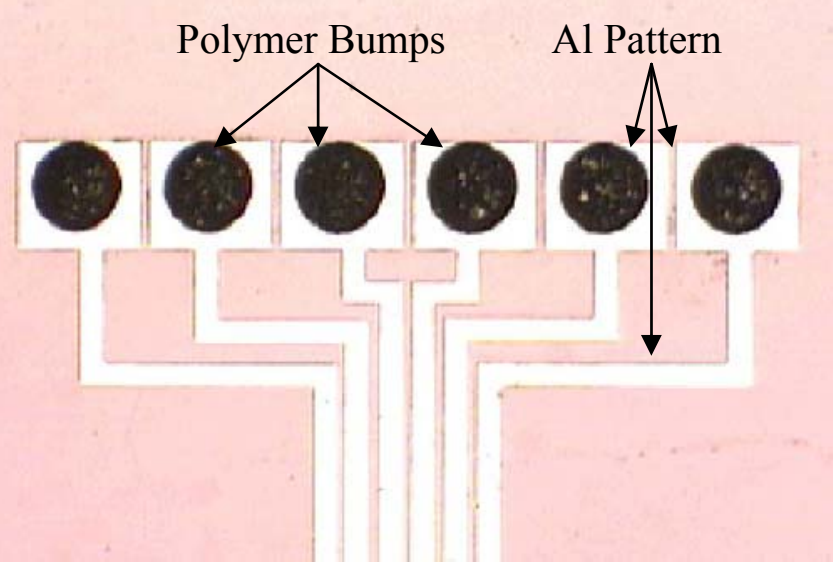

Figure 7 Polymer Bumps on MEMS wafer (100x)

\section{Evaluation of Polymer Bump Fabrication}

After removal of the PDMS mould, the polymer bumps were inspected. Two approaches were conducted for evaluation.

(A) Bump Geometry

Bump geometry was essentially a benchmark for the success of the bumping process. Bump shape was inspected by optical microscopy as well as by the surface profiler (Alpha Step 500). The measurement parameters for the profiler were set as follows:

Scan length: $500 \mu \mathrm{m}$

Scan speed: $10 \mathrm{um} / \mathrm{s}$

Stylus force: $14.5 \mathrm{~g}$

Vertical range: $300 \mu \mathrm{m}$

Vertical resolution: $250 \mathrm{~nm}$

After removal of the PDMS micro-transfer mould, the dimensions of the polymer bump are found to be typically $150 \mu \mathrm{m}$ in diameter, $92 \mu \mathrm{m}$ in height, and at a $245 \mu \mathrm{m}$ pitch The geometry of a typical bump is shown as follows:

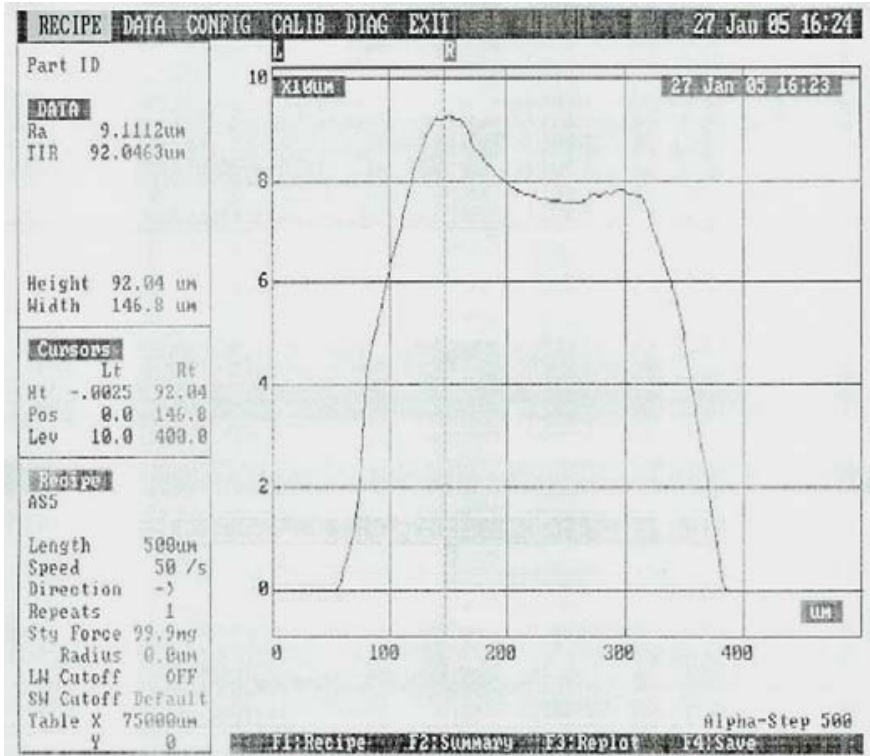

Figure 8 Sectional profile of individual bump measured by Alpha Step 500 surface profiler

10732005 Electronic Components and Technology Conference 
The sectional profile of ten bumps on one bumped chip was measured by the Alpha Step 500 to investigate the variation within one single chip. The following table shows the variation of the height of the bumps.

\begin{tabular}{|c|c|}
\hline Individual bump number & Height $(\mu \mathrm{m})$ \\
\hline 1 & 93.00 \\
\hline 2 & 91.00 \\
\hline 3 & 93.50 \\
\hline 4 & 97.50 \\
\hline 5 & 91.00 \\
\hline 6 & 91.50 \\
\hline 7 & 89.00 \\
\hline 8 & 90.00 \\
\hline 9 & 95.50 \\
\hline 10 & 92.50 \\
\hline \hline Average & 92.45 \\
\hline Standard Deviation & 2.57 \\
\hline
\end{tabular}

Table I Bump height distribution

The average height of the bumps was $92.45 \mu \mathrm{m}$ with a standard deviation of $2.57 \mu \mathrm{m}$. A lower variation of the bump height when compared with Wong et al indicates the success of the using print tool with controlled printing height.

(B) Mechanical Strength

Mechanical strength of an interconnect is a key issue in the reliability of a package, especially for long term reliability test. Shear strength was measured by Dage 4000 tester using Ball Shear Cartridge 250gram. The shear test result will evaluate the mechanical integrity of the polymer bumps. The bump shear testing parameters were set as follows:

Test Load Range: 100 gram

Shear Test Speed: $30 \mu \mathrm{m} / \mathrm{s}$

Shear height: $15 \mu \mathrm{m}$

Over travel distance: $100 \mu \mathrm{m}$

The adhesion strength of the bumps was recorded as follows:

\begin{tabular}{|c|c|}
\hline Individual bump number & Bump Strength $(\mathrm{g})$ \\
\hline 1 & 42.493 \\
\hline 2 & 43.877 \\
\hline 3 & 41.582 \\
\hline 4 & 42.834 \\
\hline 5 & 44.316 \\
\hline 6 & 43.014 \\
\hline 7 & 43.461 \\
\hline 8 & 44.779 \\
\hline 9 & 43.942 \\
\hline 10 & 43.284 \\
\hline \hline Average & 43.358 \\
\hline Standard Deviation & 0.934 \\
\hline
\end{tabular}

Table II Bump adhesion strength distribution
The average bump adhesion strength was $43.358 \mathrm{~g}$ with a standard deviation of $0.934 \mathrm{~g}$. A lower variation of the bump adhesion strength when compared with Wong et al indicates the success of the performing printing process under vacuum environment.

\section{PCB Assembly}

Flip chip process is realized as patterned die bonding on printed circuit board. A printed circuit board was customized for the flip chip process. The board was designed based on the dimension of the MEMS chip. The solder mask defined board had copper pads of $150 \mu \mathrm{m}$ diameter and copper line traces of $102 \mu \mathrm{m}$ thick. The finest copper line space was also fabricated with $102 \mu \mathrm{m}$ thick. The copper pad was plated with a thin layer of gold to prevent oxidation.

The bumped die was aligned and bonded to the PCB by curing the conductive polymer at $120^{\circ} \mathrm{C}$ inside the Toray Flip Chip Bonder. The important parameters used for the assembly process are listed as follows:

Chip Height: $0.51 \mathrm{~mm}$

Substrate (PCB) Height: $1.7 \mathrm{~mm}$

Paste pressing amount: $0.03 \mathrm{~mm}$

Paste application temperature $20^{\circ} \mathrm{C}$

Bond Head descent along Z-axis

Speed of descent $5.000 \mathrm{~mm} / \mathrm{sec}$

Start Height: $3.000 \mathrm{~mm}$

Pressing Amount: $0.05 \mathrm{~mm}$

Bond Head ascent along Z-axis

Speed of ascent: $500.000 \mathrm{~mm} / \mathrm{sec}$

End Height: $0.000 \mathrm{~mm}$

Height from contact position: $0.04 \mathrm{~mm}$

Bond Head Temperature

Standby temperature: $40^{\circ} \mathrm{C}$

Contact Temperature: $40^{\circ} \mathrm{C}$ Duration: $0.61 \mathrm{sec}$

Temperature 1: $150{ }^{\circ} \mathrm{C}$

Duration: $18 \mathrm{sec}$

Temperature $2: 220^{\circ} \mathrm{C}$

Duration: $25 \mathrm{sec}$

Temperature $3: 90^{\circ} \mathrm{C}$

Duration: $10 \mathrm{sec}$

Descending Time: $0.61 \mathrm{sec}$

Bonding Time: $53 \mathrm{sec}$

Heated bond head cooling time: $92 \mathrm{sec}$ 
The MEMS flip chip package after bonding is shown in the following figure:

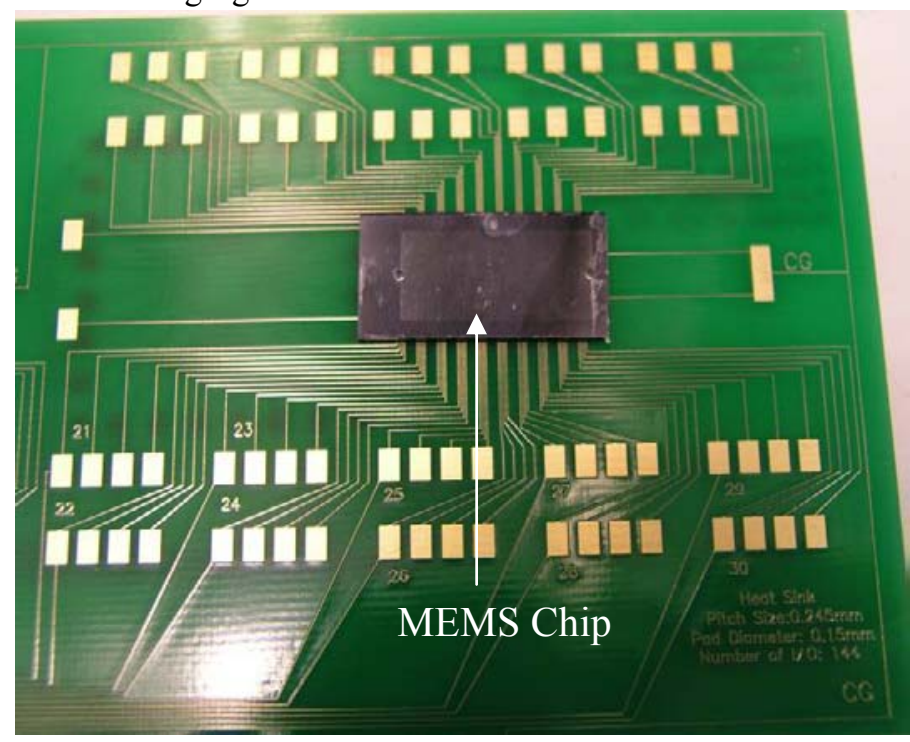

Figure 9 PCB Assembly

\section{Experimental Testing and Calibration}

Since the MEMS chip functioned only by measuring the resistance across each pair of I/Os. Electrical conductivity test was conducted after PCB assembly as a primary test for the success of packaging process. The test was conducted by measuring the resistance across a pair of I/Os using a data logger (Hewlett Packard 34970A) with a data acquisition module (Hewlett Packard 34901A). Two aluminum wires were solder to the copper pad on the PCB. One end of the wire was connected to the data acquisition module. The PCB was then put into a conventional oven. Since the glass transition temperature of the silver epoxy used for the experiment is above $80^{\circ} \mathrm{C}$ from material data sheet and the $\mathrm{Tg}$ of a typical FR-4 PCB is $125^{\circ} \mathrm{C}$. The upper limit temperature for the calibration of the MEMS chip was set at $115^{\circ} \mathrm{C}$. Resistance between two copper pads was measured with the increasing temperature. The room temperature was measured as $25^{\circ} \mathrm{C}$. A calibration curve is shown as follows:

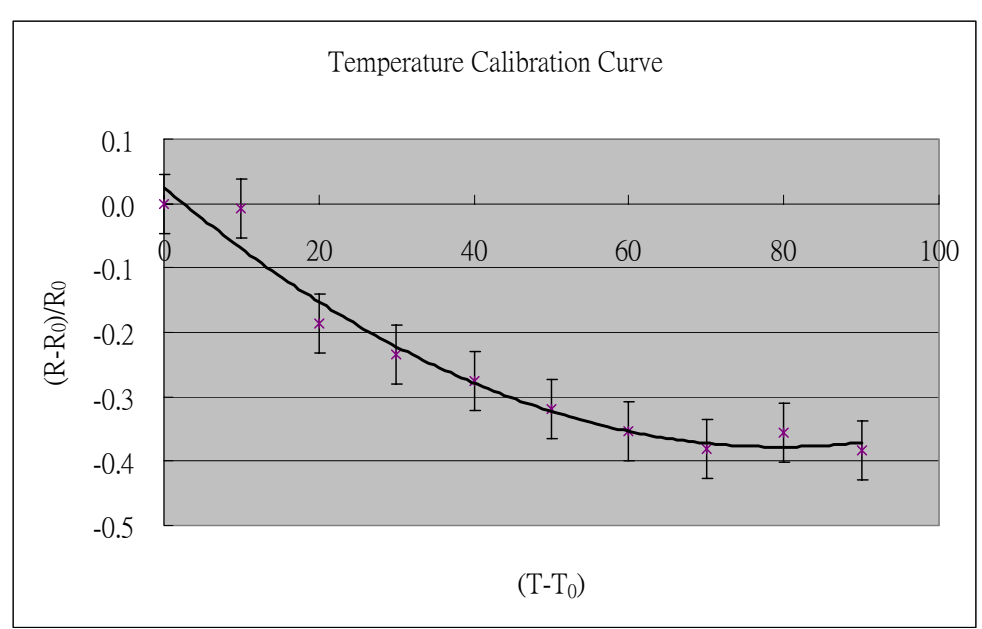

\section{Figure 10 Calibration curve of the resistance change of the} MEMS package

From Lee et al results, the MEMS package also exhibited a non-linear change of the resistance with negative increasing temperature. The package was working properly with similar trend and change of magnitude under designed temperature range.

\section{Underfill Dispensing Process}

After board assembly, samples were injected with underfill (Hysol FP4527) through a dispensing system (Camalot 1414 dispenser).

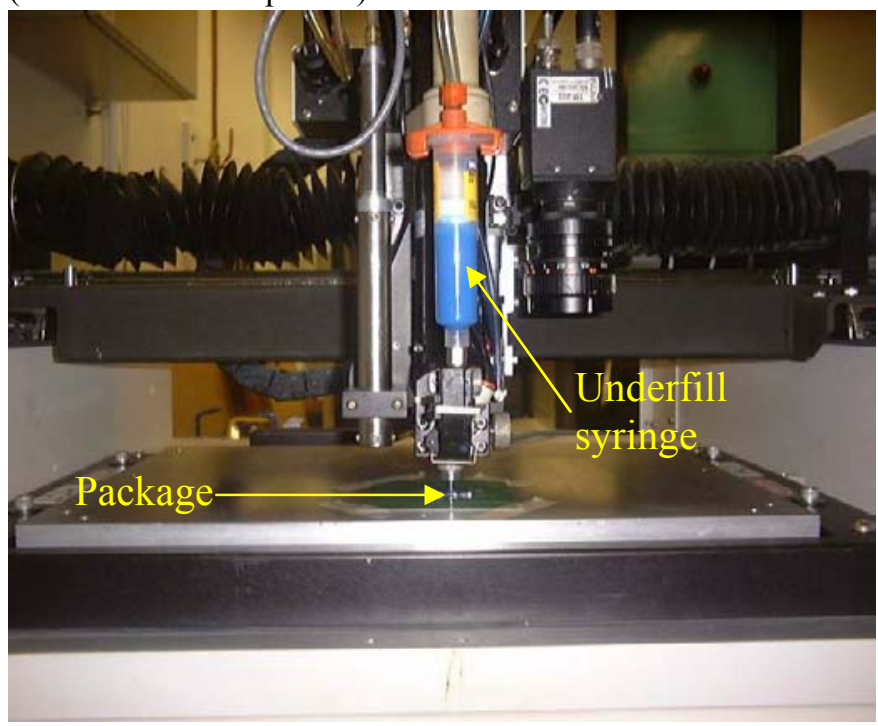

Figure 11 Underfilling process 
elow:

Needle Size: $1.28 \mathrm{~mm}$

Shoot Speed: $0.5 \mathrm{~mm} / \mathrm{s}$

Shoot Size 200 milli-second

Height of dispensing: $0.5 \mathrm{~mm}$ from PCB surface

Curing Profile: $125^{\circ} \mathrm{C}$ for $11 / 2$ hours

The package was sectioned and polished for evaluation. A cross-sectional view picture is taken as below:

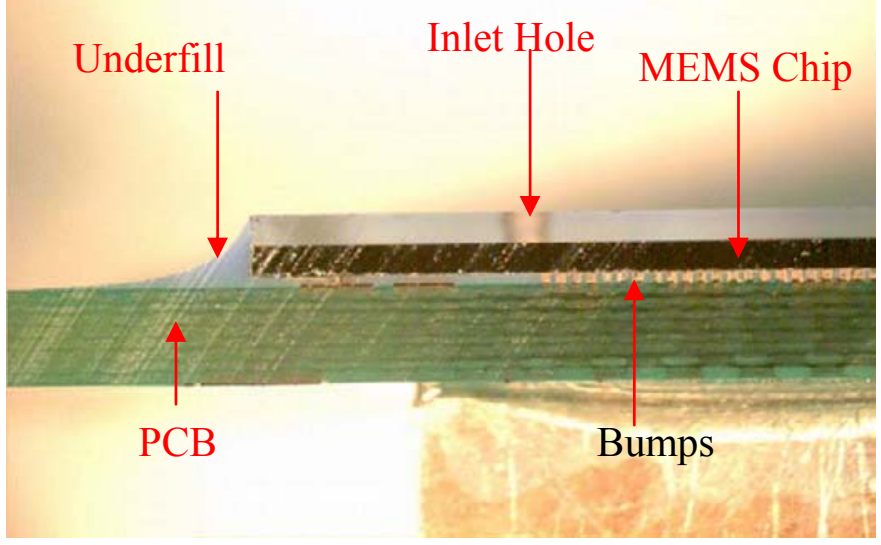

Figure 12 Cross-sectional view of the package after underfilling process $(\mathbf{8 x})$

\section{Silicon substrate}

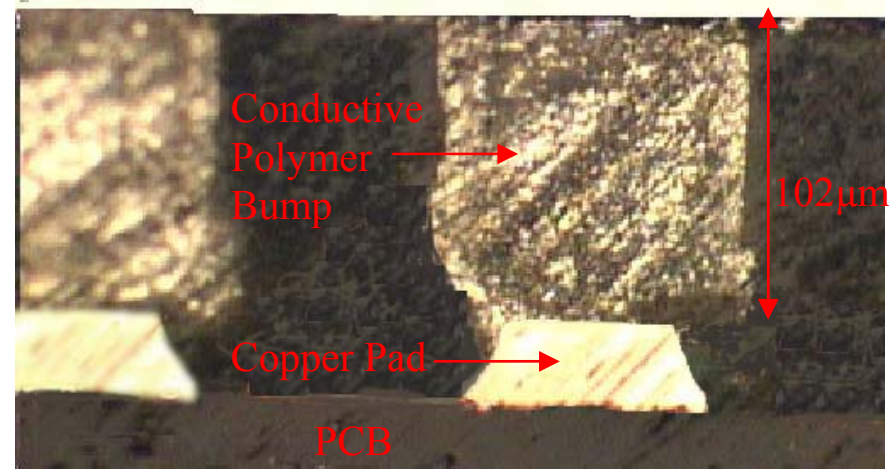

Figure 13 Cross sectional view of bumps (200x)

From cross-sectional view, the bump was well-aligned with the copper pad and each bump height was measured as $102 \mu \mathrm{m}$.

\section{Discussions}

From the results above, the variation of the geometry and the adhesion strength of the conductive polymer bumps was reduced by introducing a new printing method The vacuum printing process solved the air cushion effect in the microtransfer moulding process. From cross-sectional view of the package and the calibration curve results, it can be shown that the MEMS chip was working properly by this polymer bumping approach. The negative temperature coefficient of resistance due to phosphorus doping from the calibration curve gave a proof of the performance of the polysilicon sensor was good compared with Lee et al. The order of magnitude change in the curve is a bit lower than previous study partly because the resistivity of the conductive polymer used in this experiment is in a order of $10^{-4} \Omega-\mathrm{cm}$ which is much larger than the gold wire bonding $\left(10^{-8} \Omega-\mathrm{cm}\right)$. Nevertheless, the feasibility of this polymer bumping approach in a MEMS package is confirmed in the primary electrical conductivity testing.

\section{Conclusions}

With this polymer bumping approach, intrinsic slumping problem of the conductive polymer is resolved. A finer pitch, polymer flip chip was fabricated on MEMS by the new process. Polymer bumping process has attained a consistent performance by adopting the vacuum printing process. Although the sensitivity of the MEMS chip measured was not as high as previous research conducted. This paper addresses the feasibility of using suitable conductive polymer bumped flip chip on MEMS packages which require low temperature processing profile.

\section{Acknowledgments}

Design and fabrication of the MEMS chips was carried out by Leo M. Lee, Y.K. Lee, Yitshak Zohar. This work is supported by Hong Kong Research Grant Council grant HKUST6046/01E.

\section{References}

[1] R. H. Estes, "Process and reliability characteristics of polymer flip chip assemblies utilizing stencil printed thermosets and thermoplastics," presented at Proceedings of 3rd International Conference on Adhesive Joining and Coating Technology in Electronics Manufacturing 1998, 1998. F. W. Kulesza and R. H. Estes, "Assembling smart cards with polymer flip chip technology," Electronic Packaging Production, vol. 37, pp. 39-40, 42, 44, 1997.

C. K. Y. Wong, O. C. T. Cheung, B. Xu, and M. M. F. Yuen, "Using PDMS microtransfer molding (uTM) for polymer flip chip," presented at 53rd Electronic Components and Technology Conference, 2003.

M. Lee, Y. Y. Wong, M. Wong, and Z. Yitshak, "Size and shape effects on two-phase flow patterns in microchannel forced convection boiling," Journal of Micromechanics and Microengineering, vol. 13, pp. 155-164, 2003.

R. R.Tumula, Fundamental of Microsystems Packaging: McGraw-Hill, 2001.

K. W. Oh, C. H. Ahn, and K. P. Roenker, "Flip-chip packaging using micromachined conductive polymer bumps and alignment pedestals for MOEMS," IEEE Journal of Selected Topics in Quantum Electronics, vol. 5, pp. 119-126, 1999. 